\title{
POWER OPTIMIZATION STUDY OF A PIEZOELECTRIC ENERGY HARVESTER IN HVAC SYSTEMS BY INDUCED VORTEX SHEDDING
}

\author{
T. ANUP KUMAR ${ }^{1}$, M.V. SAI LIKHITH ${ }^{2}$, M. VENU GOPAL RAO ${ }^{3} \&$ A. SRINATH ${ }^{4}$ \\ ${ }^{1,4}$ Professor, Department of Mechanical Engineering, Koneru Lakshmaiah Education Foundation, Green Fields, \\ Vaddeswaram, Guntur, Andhra Pradesh, 522502, India \\ ${ }^{2} P G$ scholar, Department of Mechanical Engineering, Koneru Lakshmaiah Education Foundation, Green Fields, \\ Vaddeswaram, Guntur, Andhra Pradesh, 522502, India
}

${ }^{3}$ Professor, Department of Electronics and Communication Engineering, Koneru Lakshmaiah Education Foundation, Green Fields, Vaddeswaram, Guntur, Andhra Pradesh, 522502, India

\begin{abstract}
A piezoelectric PVDF film is made to vibrate in HVAC air ducts and is excited between fin attached to it and the vortices shedding from the bluff obstacle. The bluff obstacle is placed ahead of the fin and the piezo-electric film arrangement. Maximum power output from the PVDF film, is achieved when input excitations from vortex shedding are iteratively modified to match the natural frequency of the film. The incoming airflow velocity is maintained in the range of $2.5-3$ $\mathrm{m} / \mathrm{s}$. In the HVAC air duct, the output power is generated by utilizing various fin materials at an airflow speed in the range of 2.5-3 m/s. The resultant output power which could be used to power wireless sensors, is found to be in the range of $0.655 * 10^{-9} W$.
\end{abstract}

KEYWORDS: Energy Harvesting. Piezoelectric, Vortex Shedding, Wireless Sensors \& PVDF Films

Received: Feb 01, 2020; Accepted: Feb 14, 2020; Published: Mar 07, 2020; Paper Id.: IJMPERDAPR2020139

\section{INTRODUCTION}

In modern times, the energy crises has become a global issue to look for a renewable source of energy from our environment. Energy harvesting technique is an idea of converting the ambient energy into electrical energy to power local electronic devices. The significant usage of energy harvesting applications are to support wireless sensor nodes. Powering these wireless sensor nodes require long phase stability instead of short phase resolution. On micro and nanoscales, recent technologies like piezoelectric energy harvesters for advanced wireless sensor networks, biosensors and MEMS devices are being researched.

At present wireless sensor nodes are restricted by the batteries because of permanent economical limitations of restoring thousands of batteries. An energy harvesting device which are incorporate with rechargeable batteries could enhance wireless sensor nodes lifetime and reduce the usage to change the restore batteries. Over the past decade, piezoelectric energy harvesters have been increasingly investigated.

The piezoelectric effect was first introduced in 1880 by Pierre Curie and Jacques Curie. They found that if certain crystals like quartz were subjected to mechanical strain, the degree of polarization was equivalent to the applied strain and they become electrically polarized. At present, polycrystalline ceramics are commonly used piezoelectric materials. Piezoelectric ceramics like lead zicronate titanate $(\mathrm{PZT})$ and barium titanate $\left(\mathrm{BaTiO}_{3}\right)$ have high piezoelectric co-efficients. Piezoelectric polymer like polyvinylidene fluoride (PVDF) is widely used because 
of its high flexibility. PVDF films are of low cost which makes them commercially available. The piezoelectric polymer have less piezoelectric coefficient than piezoelectric ceramics.

Among vibration energy harvesters (VEH) the piezoelectric energy harvesters (PEH) are considered to be prominent. By using piezoelectric materials the mechanical energy is converted into electrical energy and this process is known as piezoelectric energy harvesting (PEH). Piezoelectric energy harvesting concentrates on harvesting low-level energy. These low-level energies are in the form of microwatts and milliwatts. Due to its convenience, low cost and small scale piezoelectric cantilevers have made them successful. In the beginning, several methods of flow-induced excitations has been analyzed. The vortices which are created by the bluff obstacle were the initial process of flow excitation which were examined. In these cases, the piezoelectric transducer generates a periodic forcing in the wake of bluff obstacle and causes it to oscillate and creates the Karman vortex street. The mechanism involved in producing the movement of the transducer, is known as vortex-induced vibration (VIV). These sort of arrangement is advantageous to analysis the optimization to tune the harvesters under flow conditions. Galloping is another aerodynamic fluctuation, is being studied which produces self-excited vibrations by a bluff obstacle which is mounted on a cantilever tip.

Xie et al.[1] proposed flow-induced vibrations to harvest energy by using a flexible piezoelectric cylinder. Onedimensional mathematical model is used to determine the performance and output power of the harvester. Under the lowest resonant frequency of the cylinder with airflow speed of $5 \mathrm{~m} / \mathrm{s}$, could generate electrical energy in the range of $103 \mathrm{~W}$ by shedding.

Hobbs and Hu [2] examined VIV to harvest wind energy through a power scavenging device of small size. The piezoelectric transducers, are made to vibrate by connecting a linear array of four cylinders in a uniform flow. Wen et al. [3] analyzed a vortex-induced vibrations (VIV) energy harvester system based on its pressure in fluid flow to optimize the harvester. A cube-shaped bluff obstacle was analyzed and it is correlated to a cylinder-shaped bluff obstacle. Under a 100 $\mathrm{k} \Omega$ resistor load with an airflow velocity of $2 \mathrm{~ms}^{-1}$ the output power of $1 \mu \mathrm{W}$ could be generated. Gao et al. [4] stated that an energy harvesting method comprising of a cylindrical expansion at the end of piezoelectric cantilever. The working principle was based on a piezoelectric beam, which is connected to the end of a cylinder is harvesting energy from the VIV phenomenon.

Dai et al. [5] recommended a theoretical model of a piezoelectric cantilever beam, connected to cylinder for energy capture. To harvest energy from vortex-induced vibrations (VIV) this design was refined based on the nonlinear parameter model. $\mathrm{Xu}-\mathrm{Xu}$ et al.[6] evaluated the vortex induced vibrations (VIV) of a circular cylinder in a dual-mass formation by energy production from the fluid flow. In the absence of the dual-mass the restrictions and advantages of the design were also presented. The improvement of efficiency of energy production had a further scope in this analysis.

Dunnmon et al. [7] developed piezoelectric patches that are fixed on a elastic strip by using an aero elastic energy harvester. The aero elastic energy harvester is installed at the end of a bluff obstacle and therefore the flapping flag was excited in an uniform axial flow. In a wind speed of $27 \mathrm{~ms}^{-1}$ they generated an output power of $2.5 \mathrm{~mW}$. Li et al. [8] investigated a model of flapping-leaf, i.e. operated based on aero elastic flapping. This type of device was suitable for lowcost organic piezo-materials. Weinstein et al. [9] proposed, by attaching a fin at the end of the piezoelectric cantilever, the VIV is excited in the steady air flow conditions to harness energy. They stated that, the airflow speeds may range from 2 to $5 \mathrm{~m} . \mathrm{s}^{-1}$ in situations of small weights that are placed along the fin. 


\section{VORTEX SHEDDING}

A vortex-induced vibrations (VIV) are produced by the bluff obstacle placed in a flowing fluid [10]. This VIV can occur with the bluff bodies and the streamlined bodies. These have been observed in various areas such as mechanical engineering, civil engineering, aerospace engineering and ocean engineering. Recently, with the revived notice of wind energy systems, oscillations of wind turbine blades draw considerations. This analysis examines the rotational analysis of a flat plate to provide a fundamental outlook of VIV in pitch oscillation in the wind tunnel.

Through the variations of fluid pressure on the structure surface, the oscillating forces are formed which are produced by vortex shedding. These vortices are scattered correspondingly from both sides of the surface when the fluid pressure fluctuates. The periodic unbalanced flow generated by the vortex shedding is distinguished as Karman vortex street.

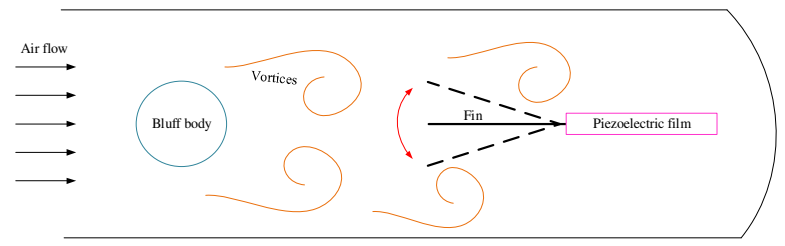

Figure 1: Schematic view of energy harvester

Usually, the flow parameter which determines the performance of the vortex shedding is distinguished by the Reynolds number which is shown in equation below

$$
R e=\frac{V_{a} D}{\vartheta}
$$

where $V_{a}$ is the velocity of the coming flow. $\mathrm{D}$ is a characteristic dimension of a body. $\vartheta$ is the kinematic viscosity of fluid.

The vortex shedding frequency has a feasible correlation with the flow velocity. These correlations are characterized by Strouhal number which is expressed as a non-dimensional parameter.

$$
S_{t}=\frac{f_{s t} D}{V}
$$

where $f_{s t}$ is the frequency of vortex shedding of a total cycle for the body at rest.

The lift and drag forces acting on the cylinder are caused by the shedding of the vortices alternately. If the cylinder is free to move the vibrations are formed in a direction horizontal to the flow which are resulted by the oscillating lift force. These types of vibrations are considered as "Vortex-Induced Vibrations".

When the vortex shedding frequency equivalent to the natural frequency of the body is known as "lock-in phenomenon" [11-13]. This is also important to identify the extent of vortex shedding frequency balancing with the fundamental structural frequency, $f_{n}$ is used to study energy harvesting device.

$$
f_{n}=\frac{1}{2 \pi} \sqrt{k / m}
$$

where, $\mathrm{k}$ is stiffness of the spring. $\mathrm{m}$ is the mass applied to the cylinder.

According to ohms law 


$$
V=I R
$$

where $\mathrm{V}$ is voltage generated by the piezoelectric harvester. $\mathrm{I}$ is amplified current supplied to the system. $\mathrm{R}$ is the resistance paired to the harvester to measure output power.

Power generated by the piezoelectric energy harvester is

$$
P=V I
$$

\section{EXPERIMENTAL SETUP}

The bluff body used in this experiment is cylindrical PVC obstacle having diameter of $1 \mathrm{~cm}$. The commercially available piezoelectric PVDF film of $25 \mathrm{~mm} \times 13 \mathrm{~mm}$ size with a thickness of $0.15 \mathrm{~mm}$ is used for energy harvesting. Polyvinylidene difluoride (PVDF) are the most regularly used piezoelectric polymers. It is a semi-crystalline polymer which is having repeating units of $\left(\mathrm{CH}_{2}-\mathrm{CF}_{2}\right)$. In an amorphous matrix approximately $50 \%$ crystals are implanted in PVDF. PVDF film is a solid polymer and it is light-weight that can be used in a broad range of thickness.

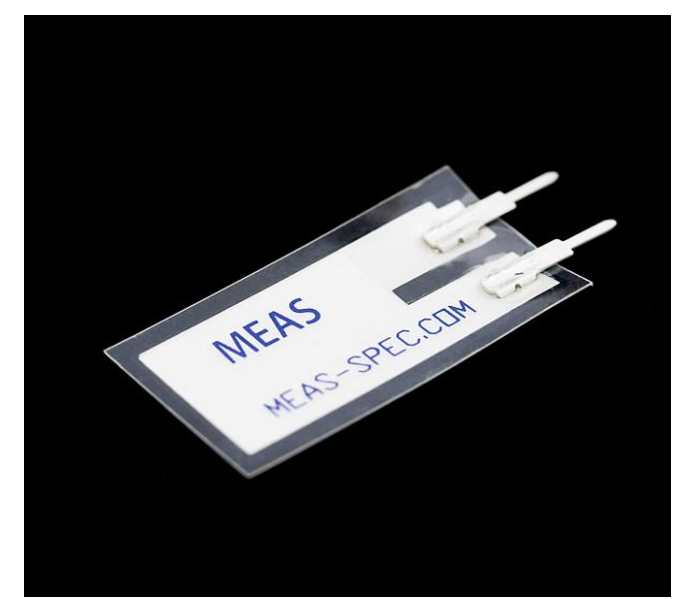

Figure 2: Piezoelectric PVDF film

Balsa wood is used as a fin material for energy harvesting because of its high stiffness to density ratio. The fin material is moulded into aerodynamic shape to allow the incoming airflow to easily flow over the fin surface. The fin location should not be too close to the cylindrical obstacle because of its inferences in the formation of vortices. Also if we place the fin too far from the cylindrical obstacle, the energy dissipates from the vortices before it reaches the film.

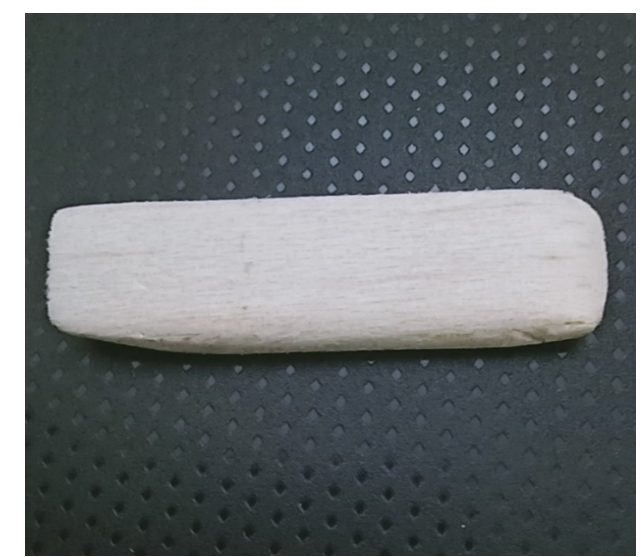

Figure 3: Fin material (Balsa wood) 
A $220 \mathrm{k} \Omega$ resistor is paired with the PVDF film to measure power output. The other end of the resistor is connected to the digital oscilloscope which is used to measure the resonant frequency. The incoming air velocity is measured using a digital anemometer.

\section{EXPERIMENTAL WORK}

In HVAC air ducts, the PVDF film is made to vibrate and is excited between fin attached to it and the vortices shedding from the bluff obstacle. The bluff obstacle is made of cylindrical body. The incoming air is allowed to pass through the bluff obstacle. The air flowing past the bluff obstacle creates a periodic oscillating field behind the obstacle and thus phenomenon is referred to as Karman vortex street.

The vortices shedding from the bluff obstacle exerts a force on the both sides of the fin which makes them vibrate in to and fro motion. This vibrating fin is attached to the piezoelectric PVDF film which is subjected to deformation. Due to this, deformation polarization occurs over the piezoelectric material and voltage is induced. In this experiment, two types of fin materials namely cardboard and balsa wood respectively are used to harness the energy from the harvester.

\section{RESULTS}

An experiment is conducted by using fin materials like cardboard and balsa wood at an airflow speed in the range of 2.5-3 $\mathrm{m} / \mathrm{s}$. The $220 \mathrm{k} \Omega$ resistor which is paired to the harvester's one end and the other end connected to the digital oscilloscope gives the resonant frequency at a flow speed of $2.5-3 \mathrm{~m} / \mathrm{s}$. By using these resonant frequencies, the maximum output power is found out to be

Table 1: Experimental readings of energy harvester

\begin{tabular}{|l|c|c|}
\hline \multicolumn{1}{|c|}{ Fin Material } & Voltage generated(V) & Output Power(W) \\
\hline Card board & $34.40 \mathrm{mV}$ & $5.38 * 10^{-9} \mathrm{~W}$ \\
\hline Balsa wood & $12.00 \mathrm{mV}$ & $0.655 * 10^{-9} \mathrm{~W}$ \\
\hline
\end{tabular}

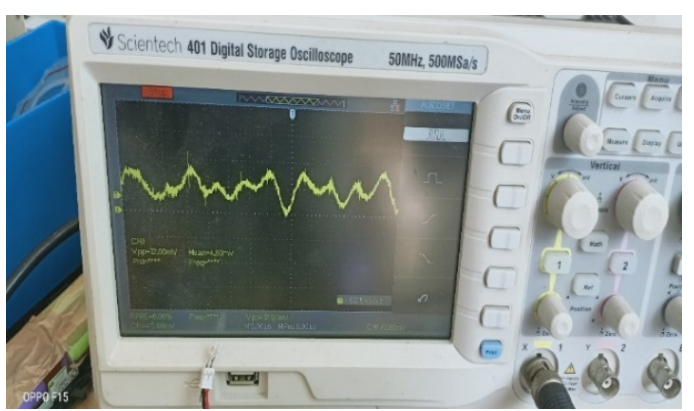

Figure 4:Carboard reading shown in digital anemometer 


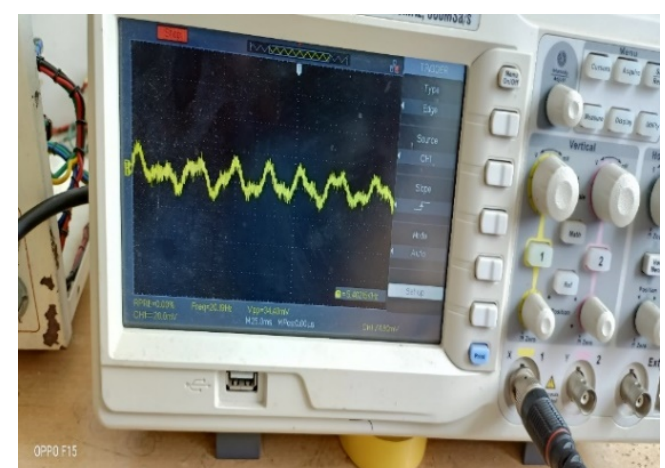

Figure 5: Balsa wood reading shown in digital anemometer.

We had conducted experiment by using PVDF film containing no mass as a piezoelectric bender. The fin material is made up of balsa wood shaped aerodynamically to avoid the disturbance of air flow and allows it to easy flow on the surfaces of the fin material. We can also conduct the experiment by using a flow straightener placed ahead of the piezoelectric harvester to decrease turbulence of the incoming air flow In the future study by using this experimental setup we can improve the power output by making slight modifications. The PVDF film can be clamped in both horizontal and vertical direction of airflow. The fin material is made up of plastic or polymer in the shape of triangle and it is attached to the free end of the PVDF film through a plastic hinge.

\section{CONCLUSIONS}

In this study, an experimental setup is installed that can harvest available vibration energy into electrical energy to measure maximum output power by using different fin materials at a flow speed of $2.5-3 \mathrm{~m} / \mathrm{s}$. By adding fin material to the tip of the PVDF film, there will be an increase in output power generation. The power generated at an airflow speed of $2.5-3 \mathrm{~m} / \mathrm{s}$ by the cardboard material is $5.38 \mathrm{nW}$ and the power generated at an airflow speed of $2.5-3 \mathrm{~m} / \mathrm{s}$ by the balsa wood is 0.655 $\mathrm{nW}$. The resultant output power is sufficient to power a wireless sensor node.

\section{REFERENCES}

1. J. Xie, J. Yang, H. Hu, Y. Hu, X. Chen, A piezoelectric energy harvester based on flow-induced flexural vibration of a circular cylinder, J. Intell. Mater. Syst. Struct. 23 (2012) 135e139, https://doi.org/10.1177/1045389x11431744

2. W.B. Hobbs, D.L. Hu, Tree-inspired piezoelectric energy harvesting, J. Fluids Struct. 28 (2012) 103e114, https://doi.org/10.1016/j.jfluidstructs.2011.08.005

3. Q. Wen, R. Schulze, D. Billep, T. Otto, T. Gessner, Modeling and optimization of a vortex induced vibration fluid kinetic energy harvester, Procedia Eng. 87 (2014) 779e782, https://doi.org/10.1016/i.proeng.2014.11.656

4. X. Gao, W.-H. Shih, W.Y. Shih, Flow energy harvesting using piezoelectric cantilevers with cylindrical extension, IEEE Trans. Ind. Electron. 60 (2013) 1116e1118, https://doi.org/10.1109/tie.2012.2187413

5. H.L. Dai, A. Abdelkefi, L. Wang, Theoretical modeling and nonlinear analysis of piezoelectric energy harvesting from vortexinduced vibrations, J. Intell. Mater. Syst. Struct. 25 (2014)1861e1874.https://doi.org/10.1177/1045389X14538329

6. H.D. Akaydin, N. Elvin, Y. Andreopoulos, The performance of a self-excited fluidic energy harvester, Smart Mater. Struct. 21 (2012) 25007.https://doi.org/10.1088/0964-1726/21/2/025007

7. J.A.aDunnmon, S.C.aStanton, B.P. Mann, E.H. Dowell, Power extractionafromaaeroelastic limitacycle oscillations, J. Fluids Struct. 27a(2011) 1182e1198a 
8. S. Li, J. aYuan, H. aLipson, Ambient wind energy harvesting usingacross-flowafluttering, J. Appl. aPhys. 109 (2011) 2e5, https://doi.org/10.1063/1.3525045.

9. L.A. Weinstein, M.R. Cacan, aP.M. aSo, P.K. Wright, Vortex sheddingainducedaenergyaharvesting from piezoelectric materialsain heating, ventilation and air conditioning flows, SmartaMater. aStruct. 21a(2012) 45003, https://doi.org/10.1088/0964-1726/21/4/045003

10. Neha Yadavaand PrashantaBaredar, A review on piezoelectricaenergy harvestingasystemausingavortex induced vibration, AIPaConferenceaProceedings 2039, 020054 (2018); doi: 10.1063/1.5079013

11. Antiopi-Malvina Stamatelloua, AnestisaI. Kalfas, Testing of piezoelectric energyaharvestersaisolated from base vibrations, EnergyaConversionaandaManagement 196 (2019)a717-728, https://doi.org/10.1016/j.enconman.2019.06.04

12. Metta,aV.R., Konijeti, R., Dasore, A., Thermal design of spiral plateaheataexchanger throughanumerical modelling, InternationalaJournal ofaMechanical Engineering and Technology, 9(2018) 736-745, JMET_09_07_077

13. Ganna, S., Konijeti, aR., Dasore, aA., Comparativeastudyaof physical and chemical adsorbents with compositeaadsorbentapairs foraapplication inaadsorption refrigeration system, InternationalaJournal ofaMechanical Engineering andaTechnologya, 9(2018) a, 702-711,_IJMET_09_07_073 

